\title{
The Effect of Respiration-Induced Heart Movements on the ECG
}

\author{
RAMÓN PALLÁS-ARENY, SENIOR MEMBER, IEEE, JOSEP COLOMINAS-BALAGUÉ, \\ STUDENT MEMBER, IEEE, AND F. JAVIER ROSELL, STUDENT MEMBER, IEEE
}

\begin{abstract}
We present a model describing the rotation of the cardiac vector as a possible mechanism resulting in the presence of respiratory information in the ECG. The way in which this information is revealed is analyzed and the predictions subjected to qualitative experimental assessment via spectral analysis.

The results show that respiratory frequencies occur in the ECG spectrum due to heart movement. By measuring on a patient wearing a pacemaker and ventilated to control respiratory rate we show that even in the absence of respiratory sinus arrhythmia (RSA) there is a baseband information in the ECG spectrum, attributable neither to electrode artifacts nor to emg, and sidebands from the respiratory cycle.
\end{abstract}

\section{INTRODUCTION}

$\mathrm{T}$ HE ECG has been considered a respiratory information-carrying signal by some authors, mostly because of respiratory sinus arrhythmia (RSA) [1]. But given the proximity between lungs and heart, the measurements carried out in the respiratory and cardiovascular systems are also subjected to the physical influences of the other. When recording the ECG, for example, efforts are usually made to reduce these interactions or their consequences.

On the other hand, when analyzing heart rate variability spectral methods are normally used without taking into account any respiratory effect other than RSA. Some authors analyze the cardiac rate signal [2], but others analyze the ECG [3] or the pulse wave [4], [5].

We present a model describing how a rotation of the cardiac vector introduces respiratory information into the ECG. After considering the effects of respiratory sinus arrhythmia (RSA), the results obtained in the qualitative experimental assessment of this model are also discussed. Our aim is to show that heart movements produce respiratory frequencies in the ECG spectrum similar to those resulting from RSA.

\section{Effects of Respiration on the Frontal ECG}

The effects on the ECG of heart displacements produced by respiratory movements were first systematically analyzed by Einthoven et al. [6]. In this classic paper, most of the changes in ECG amplitude and waveform were

Manuscript received December 28, 1987; revised October 17, 1988 This work was supported in part by the Comisión Asesora de Investigacion Cientifica y Tecnica Project 2671-83.

The authors are with the Division de Instrumentacion y Bioingenieria, Departamento de Ingenieria Electronica, 08080 Barcelona, Spain.

IEEE Log Number 8826259 attributed to these movements and to respiratory-induced cardiac rhythm variations. These effects were most noticeable in the standard II lead. Nowadays it is a wellknown fact that respiratory action produces a rotation of the cardiac vector and the effect by the respiratory sinus arrhythmia on the ECG signal is also well known.

In order to describe the result of this interaction, a first step is to consider the projection of the cardiac vector on the frontal plane and a rotation round an axis perpendicular to it. If the inclination of the cardiac vector with respect to lead I is $\alpha$, the voltage measured on this lead, for example, will be

$$
V_{l}=V \cos \alpha
$$

If the ECG is considered to be a periodic signal and if, at an initial stage, the periodic rotation produced by respiration is assumed to be sinusoidal, $V_{I}$ can then be expressed as

$$
\begin{aligned}
V_{I}= & \left(\sum_{n} V_{n} \cos \left(n \omega_{c} t+\Phi_{n}\right)\right) \\
& \cdot\left(\cos \left(\alpha_{0}+\Delta \alpha \cos \omega_{r} t\right)\right)
\end{aligned}
$$

where $\omega_{c}$ is the cardiac pulsation, $\omega_{r}$ is the respiratory pulsation, and $\Delta \alpha$ is the maximum angular displacement caused by the respiratory action. The second cosine term for (2) can be expressed as a product of circular functions

$$
\begin{aligned}
\cos \left(\alpha_{0}+\Delta \alpha \cos \omega_{r} t\right)= & \cos \alpha_{0} \cdot \cos \left(\Delta \alpha \cos \omega_{r} t\right) \\
& -\sin \alpha_{0} \cdot \sin \left(\Delta \alpha \cos \omega_{r} t\right)
\end{aligned}
$$

Whenever $\Delta \alpha<<\pi / 2$, the sine and cosine functions can be approximated by the first terms of their series development. In our case-and according to Ruttkay-Nedecký [7] and Beswick and Jordan [8] $-\Delta \alpha=10^{\circ}$ is an acceptable value. Therefore we have

$$
\begin{aligned}
& \cos \left(\Delta \alpha \cos \omega_{r} t\right) \approx 1-\frac{(\Delta \alpha)^{2}}{2}\left(\frac{1}{2}+\frac{1}{2} \cos 2 \omega_{,} t\right) \\
& \sin \left(\Delta \alpha \cos \omega_{r} t\right) \approx \Delta \alpha \cdot \cos \omega_{r} t .
\end{aligned}
$$

By substituting (4) and (5) in (3), and if second-order terms in $\Delta \alpha$ are considered small enough to be disre- 
garded, the result is

$$
\begin{aligned}
V_{I}= & \left(\cos \alpha_{0}\right) \sum_{n} V_{n} \cdot \cos \left(n \omega_{c} t+\Phi_{n}\right) \\
& +\left(\Delta \alpha \cdot \sin \alpha_{0}\right)(1 / 2) \sum_{n} V_{n} \\
& \left.\cdot \cos \left(\left(n \omega_{c} \pm \omega_{r}\right) t+\Phi_{n}\right)\right)
\end{aligned}
$$

The interpretation is now a simple one. As a consequence of the periodic rotation of the cardiac vector, the spectrum of the resulting signal consists of the initial one plus a set of lateral bands at each side of the cardiac harmonics located at a distance determined by the respiratory frequency. If the ECG has a direct component $(n=0)$, the movement described by the cardiac vector appears as a baseband signal. Depending on the frequency response of the electrocardiograph, this component will undergo a more or less pronounced attenuation.

The respiratory-induced movement is probably not sinusoidal and the analytical expression of the real ECG spectrum will thus be more involved. One clue to it is obtained by considering that the "distorted" ECG can be described by the product of two temporal functions, one associated with the respiration, the other with the cardiac activity, in a manner similar to that indicated by (2). The ECG spectrum can therefore be obtained by the convolution of the corresponding frequency spectra of the temporal functions. The result can be interpreted as if the respiratory signal had been sampled by the cardiac signal. According to our simplified interpretation, the ECG spectrum will have a baseband information (respiratory signal) and a lateral band at each side of the cardiac rhythm and its harmonics. In other words, the result obtained for a sinusoidal rotation can be extended to other periodic rotations.

The preceding model does not include the linear displacement of the cardiac vector. Using a theoretical eccentric spheres model, Rudy et al. [9] showed that an 1 $\mathrm{cm}$ variation in the location of the heart produces a 24.2 percent variation in surface potential. This change in amplitude will also have a repercussion on the ECG spectrum. Its effect can be analyzed by considering that the "distorted" ECG can again be described by the product of two temporal functions. The spectrum, therefore, will be similar to that described above.

One way of checking whether or not the modulation described by (6) in fact takes place is by means of ECG spectral analysis. Yet in doing so, other factors producing spectral modulation must be taken into account. One of these factors is the variability of the cardiac rhythm, in particular as a result of respiration.

In [10] a method is described for enhancing the infracardiac frequency content of cardiovascular signals, in particular pulse and flow. This consists of a frequency modulation of the cardiac rhythm by a periodic signal of lower frequency. They show that the new spectral components are those of the modulating signal. Thus, a res- piratory-induced variation of the cardiac rhythm should be revealed by the presence in the ECG of the respiratory rhythm and its harmonics.

In a rather different work [11], Rompelman et al. studied the amplitude spectrum of an event series whose frequency depended on a modulating periodic signal. This spectrum included components proportional to the corresponding components of the modulating signal and the harmonics of the unmodulated pulse repetition frequency, accompanied by sum and difference components of this frequency and that of the modulating signal.

Thus, in the case of a respiratory sinus arrhythmia, a spectral analysis of the ECG should reveal a baseband content determined by the respiratory frequency and its harmonics, and lateral bands at each side of the cardiac frequency and its harmonics, located at a distance determined by the respiratory rhythm.

The measurements carried out in a normal situation are subject to the variability of the respiratory rhythm itself. Its effect on the ECG spectrum through its influence on the cardiac rhythm will therefore be more complex than is indicated by the above-mentioned analysis. In fact, Richardson et al. [3] showed that "in the case when the fundamental frequency (of the ECG) is a random variable (frequency modulation) with a Gaussian distribution, the comb structure of the power spectrum of the original periodic model gets smoothed out, leaving a broadened bellshaped structure at each location of the tooth instead of a discrete impulse."

\section{Experimental Results and Discussion}

The model we have derived in Section II suggests that a spectral analysis of the ECG signal should include a baseband content and sidebands on each cardiac harmonic determined by the respiration.

In order to verify, to a certain extent, whether heart movements result in the presence of respiratory information in the ECG, we have carried out measurements. In order to eliminate the foreseeable consequences of cardiac rhythm variability, a patient wearing a pacemaker was selected. Although it was a demand pacemaker, during the recording there was no spontaneous cardiac activity, the cardiac rhythm thus being well known $(2.05 \mathrm{~Hz})$. For better identification of the presence of respiratory information, we decided to measure on a patient who at the same time was undergoing artificial ventilation. The respiratory frequency had been set at $0.3 \mathrm{~Hz}$.

The ECG was recorded using a magnetic recorder connected to a Cardioline electrocardiograph, model eta 150. The electrodes for the standard leads were placed at the extremities so that there were no respiratory-induced movements, and no diaphragm emg. The signal was analyzed off-line using a Hewlett Packard 3582A Spectrum Analyzer.

For the standard III lead, which amplitude was intermediate with respect to those of leads I and II, the spec- 

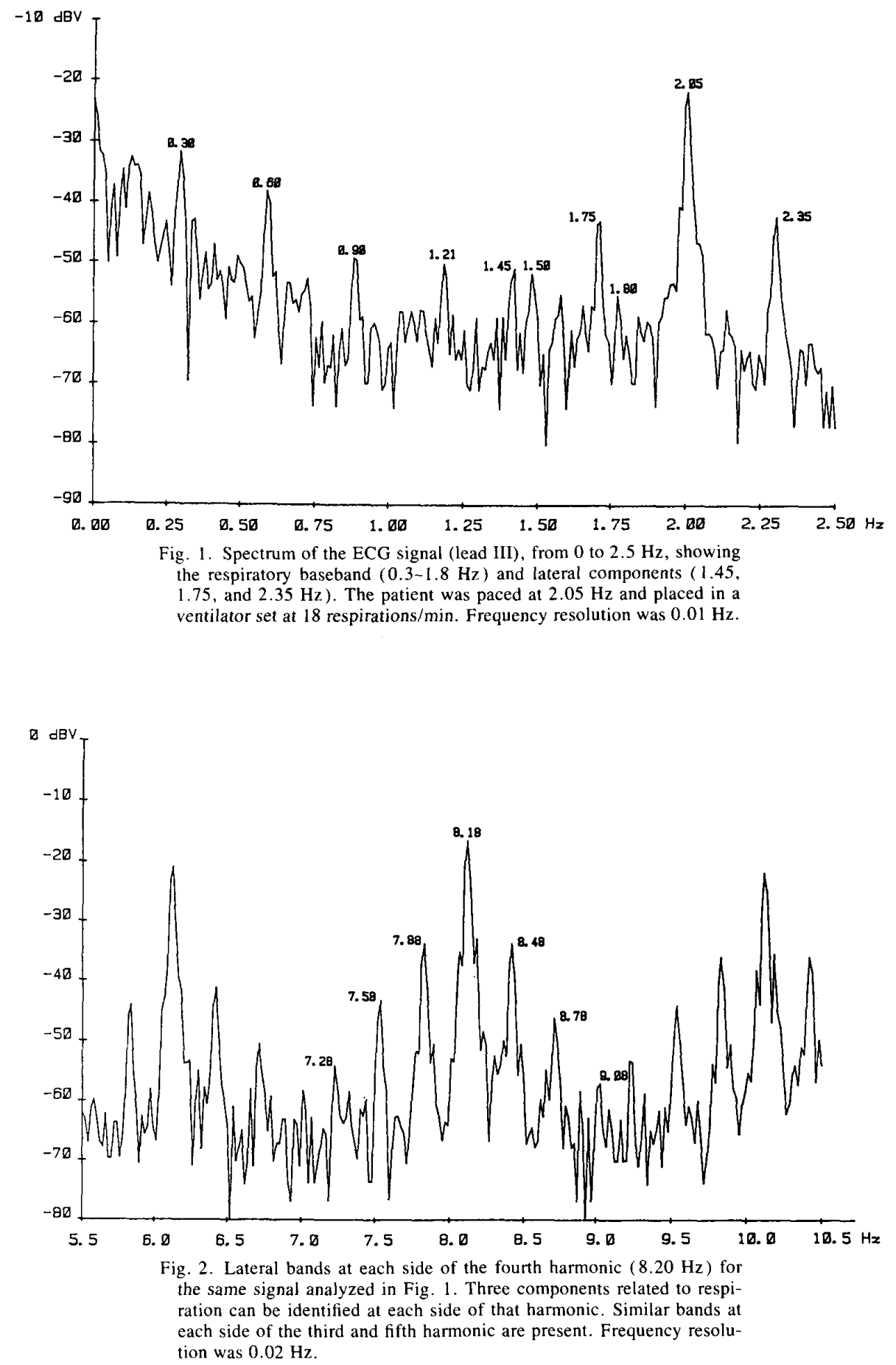

trum shown in Fig. 1 was obtained in the band from 0 to $2.5 \mathrm{~Hz}$, using a Hanning window. Leads I and II showed the same bands with different amplitudes. The presence of respiration harmonics from 0.3 to $1.8 \mathrm{~Hz}$ is apparent. The components at 1.75 and $2.35 \mathrm{~Hz}$ are located at 0.3
$\mathrm{Hz}$ from the cardiac rhythm $(2.05 \mathrm{~Hz})$ and there is also a component at $1.45 \mathrm{~Hz}$; that is to say, at $0.6 \mathrm{~Hz}$ below this rhythm. This is completely in accordance with (6).

The presence of lateral bands at each side of the cardiac harmonics can be better assessed by an analysis of the 

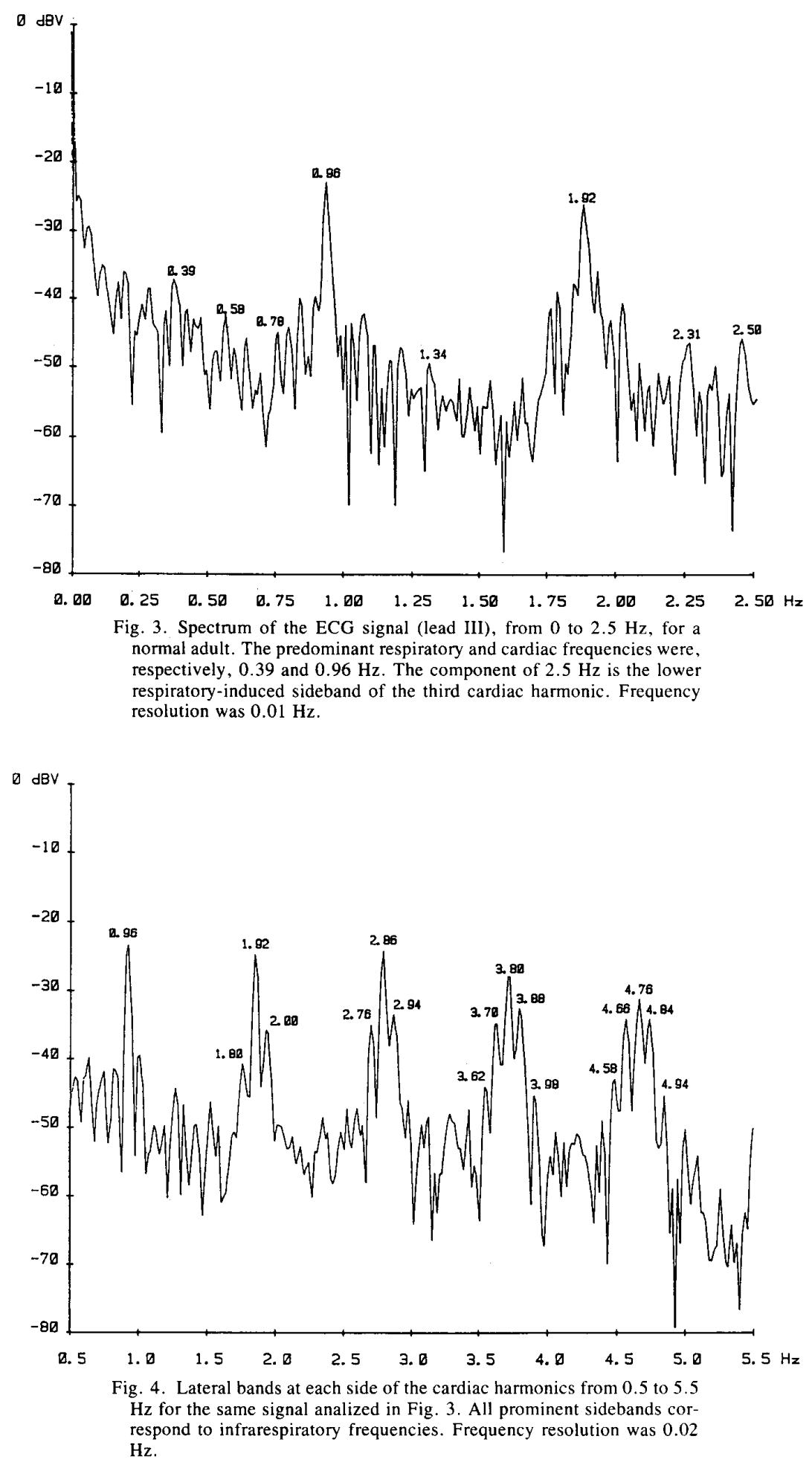

frequency components with higher amplitude. A scanning of the entire spectrum showed that the fourth harmonic was the most suitable one. The spectrum obtained in the band from 5.5 to $10.5 \mathrm{~Hz}$ for the very same signal whose baseband is the one shown in Fig. 1 is presented in Fig. 2. The presence of bands at each side of the $8.2 \mathrm{~Hz} \mathrm{com}$ - 
ponent, placed at $0.3,0.6$, and $0.9 \mathrm{~Hz}$ apart is quite clear. Similar bands can be seen at each side of the 6.15 and $10.25 \mathrm{~Hz}$ components of the ECG. There is a complete frequency symmetry, as predicted by (6).

The cardiac rhythm being constant, the presence of baseband information can in principle be attributed, at least in part, to the displacement of the heart vector. In any case, it is true that there is respiratory information in addition to that provided by the RSA, because in Figs. 1 and 2 the patient had a fixed pacing rhythm. Furthermore, even in the absence of RSA, the distortion of the ECG produced by the respiration is important if judged from the spectral analysis. The movement of the cardiac generator as described by our model can explain the presence of components in the spectrum of the ECG that are not harmonics of the cardiac rhythm.

The spectrum for normals is complex on account of the variability in heart and respiratory rhythms. Fig. 3 shows the spectrum corresponding to lead III in a healthy subject. Certain sidebands related to the main respiratory component of $0.39 \mathrm{~Hz}$ can be measured on either side of some cardiac harmonics. The band at $2.5 \mathrm{~Hz}$ corresponds to $3 \times 0.96-0.38 \mathrm{~Hz}$, that is to say, the lower respiratory band from the third cardiac harmonic (shown in Fig. 4 at $2.86 \pm 0.02 \mathrm{~Hz}$ ).

When comparing with Fig. 1, it can be noted that in Fig. 3 there are some sidebands in addition to the respiratory ones. Also that the respiratory rhythm itself produces a broad band-around $0.39 \mathrm{~Hz}$-because of its own variability. In Fig. 3, therefore, it is impossible to say whether the bands produced by the respiration are due to heart movements or to RSA.

Fig. 4 shows the spectrum from 0.5 to $5.5 \mathrm{~Hz}$ for the same ECG signal analized in Fig. 3. Now it is even difficult to identify respiratory-induced sidebands similar to those in Fig. 2. By contrast, some components placed at 0.8 to $0.12 \mathrm{~Hz}$ from the cardiac harmonics can clearly be distinguished. According to the model by Rompelman et al. [3] these bands can be linked to heart rate variability. But, according to the same model, the presence of bands from 0.8 to $0.12 \mathrm{~Hz}$ in Fig. 3 can either be a result of heart rate or respiratory rate variability.

\section{Conclusions}

By using a simple model we have shown how heart displacement can result in the presence of respiratory information in the ECG. Some easily recognizable effects of this interaction are a presence of a baseband and lateral bands at each side of the cardiac harmonics in the ECG spectrum. The description of the consequences of these interactions brings new information which is useful when analyzing the ECG signal.

For example, the experimental results indicate that a spectral analysis of the ECG signal intended to study the respiratory sinus arrhythmia [5] may lead to the wrong conclusions because the mechanical action of the respiration on the ECG results in the same kind of frequency bands as those of the RSA.

From a different point of view, it can also be concluded that just as signals modulating the cardiac rhythm reveal themselves in the ECG spectrum as baseband components, signals modulating the respiratory rhythm should also be apparent in the same spectrum. Therefore, whenever the respiratory frequency is not constant, an analysis of the presence of infrarespiratory components in the spectrum of the ECG, or, for that matter, of any other cardiovascular signal, should take this effect into account.

Finally, if a mechanical interaction between the respiratory activity and arterial pressure is considered, the results discussed above for the spectrum of the ECG can also be applied to the pulse wave. Kitney et al. [5] observed that a respiratory component was present within the blood pressure signal despite fixed rate cardiac pacing. By applying our results, their observation has a straightforward explanation.

\section{ACKNOWLEDGMENT}

The authors are deeply indebted to Dr. A. M. Muñoz, Head of Cardiology at the Hospital General Nuestra Sra. del Mar in Barcelona for his advice, help, and encouragement during the experimental part of this work. The authors appreciate many useful comments and suggestions made by two anonymous reviewers.

\section{REFERENCES}

[1] B. F. Womack, "The analysis of respiratory sinus arrhythmia using spectral analysis and digital filtering," IEEE Trans. Biomed. Eng., vol. BME-18, pp. 399-409, 1971.

[2] O. Rompelman, J. B. I. M. Snijders, and C. J. Van Spronsen, “The measurement of heart rate variability with the help of a personal computer," IEEE Trans. Biomed. Eng., vol. BME-29, pp. 503-510, 1982

[3] J. M. Richardson, V. K. Murthy, and L. J. Haywood, "Effect of random frequency modulation on ECG power spectra," IEEE Trans. Biomed. Eng., vol. BME-26, pp. 109-112, 1979.

[4] W. Craelius, V. K. H. Chen, M. Restivo, and N. El-Sherif, "Rhythm analysis of arterial blood pressure,"' IEEE Trans. Biomed. Eng., vol BME-33, pp. 1166-1172, 1986.

[5] R. I. Kitney, T. Fulton, A. H. McDonald, and D. A. Linkens, "Transient interactions between blood pressure, respiration and heart rate in man," J. Biomed. Eng., vol. 7, pp. 217-224, 1985.

[6] W. Einthoven, G. Fahr, and A. de Waart, "On the direction and manifest size of the variations of potential in the human heart and on the influence of the position of the heart on the form of the electrocardiogram," Pfluger's Arch.f. d. ges. Physiol., vol. 150, pp. 275315,1913 . English translation by H. E. Hoff and P. Sekelj, Amer. Heart J., vol. 40, pp. 163-193, 1950.

[7] I. Ruttkay-Nedecký, "Effects of respiration and heart position on the cardiac electric field," in The Theoretical Basis of Electrocardiography, C. V. Nelson and D. B. Geselowitz, Eds. Oxford: Clarendon Press, 1976, pp. 120-134.

[8] F. W. Beswick and R. C. Jordan, "Effect of expiratory and inspiratory breath-holding on the lead-field spatial vectorcardiogram," Amer. Heart J., vol. 62, pp. 379-390, 1961.

[9] Y. Rudy, R. Plonsey, and J. Liebman, "The effects of variations in conductivity and geometrical parameters on the electrocardiogram, using an eccentric spheres model,' Circ. Res., vol. 44, pp. 104-111. 
[10] J. A. Ringo, K. B. Campbell, N. S. Peterson, and R. A. Baker, "Frequency modulation of heart rate to increase low-frequency content of cardiovascular signals," IEEE Trans. Biomed. Eng., vol. BME-29, pp. 537-541, 1982 .

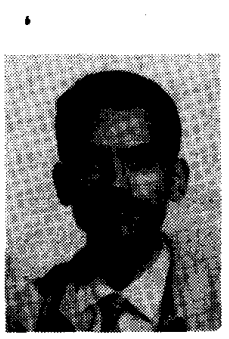

Ramón Pallás-Areny (M'81-SM'88) received the degrees of Ingeniero Industrial and Doctor Ingeniero Industrial in 1975 and 1982 , respectively, both from the Politechnic University of Catalonia, Barcelona, Spain.

$\mathrm{He}$ is currently Professor of Bioengineering, Electronic Instrumentation and Transducers, and Head of the Instrumentation and Bioengineering Division of the Department of Electronic Engineering at the same University. His present research interests are high resolution ECG and physiological measurements based on electrical impedance methods. He is author of Basic Electronic Instruments (1987) and Transducers and Signal Conditionning (1989), and coauthor of An Introduction to Bioengineering (1988), all published in Spanish by Marcombo (Barcelona, Spain).

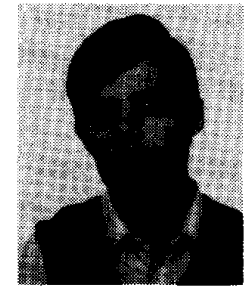

Josep Colominas-Balagué (S'85) received the degree of Ingeniero de Telecomunicación from the Politechnic University of Catalonia, Barcelona, Spain, in 1985.

$\mathrm{He}$ is currently Assistant Professor at the Department of Electronic Engineering where he is working towards the $\mathrm{Ph}$. D. degree. His major research interests are high resolution ECG and bioelectric signal processing.

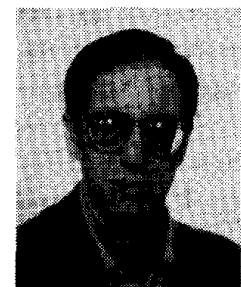

F. Javier Rosell (S'84) received the degree of Ingeniero de Telecomunicación from the Politechnic University of Catalonia, Barcelona, Spain, in 1983.

$\mathrm{He}$ is currently Assistant Professor at the Department of Electronic Engineering where he is working towards the Ph.D. degree. His major research interest is physiological measurements based on electrical impedance methods, in particular electrical impedance imaging. 